\title{
A Spectral Approach to Peak Velocity Estimation of Pipe Flows from Noisy Image Sequences
}

\author{
Ecaterina Bodnariuc
}

\begin{abstract}
Motivated by plane wave ultrasound image velocimetry (a.k.a. Echo PIV), we introduce a novel method to globally estimate the velocity field of a laminar and steady flow from the motion of tracer particles. Our approach exploits the fact that the equation of motion for pipe flows of constant circular cross-section is governed by a single global but unknown parameter. We connect this parameter to the Fourier spectrum of the input image sequence of the tracer particles and formulate our motion estimation problem as a spectral support estimation problem. The approach is validated on both simulated and experimental real data. It returns accurate velocity estimates after few seconds runtime and effectively copes with speckle patterns and noise.
\end{abstract}

\section{Introduction}

Our work is motivated by the task of estimating the instantaneous velocity of vessel blood flow using plane wave ultrasound image velocimetry (a.k.a. Echo PIV) $\left[\mathrm{LBE}^{+} 15, \mathrm{VKV}^{+} 16, \mathrm{CPTP} 16\right]$. Ultrasound techniques are widely used to measure blood flow in clinical applications. They enable noninvasive measurements that can be applied to opaque flows. Additionally, the use of plane wave ultrasound imaging enables attainment of very fast frame rates (larger than 1000 frames per second) over a large field of view and improves the temporal resolution of the signal [TF14].

Echo PIV is a velocimetry technique that applies optical PIV analysis algorithms to sequential ultrasound images and has been developed to improve blood flow analysis using clinical ultrasound machines [KHSO4, Poe17]. Schematic representation of Echo PIV is shown in Fig. 1.1. The presented work is motivated by the application

Key Words: Harmonic image sequence analysis, image motion estimation, Echo PIV.

2010 Mathematics Subject Classification: Primary 92C55, 42A38; Secondary 83C10, 90C90.

Received: April, 2017.

Revised: July, 2017.

Accepted: July, 2017. 

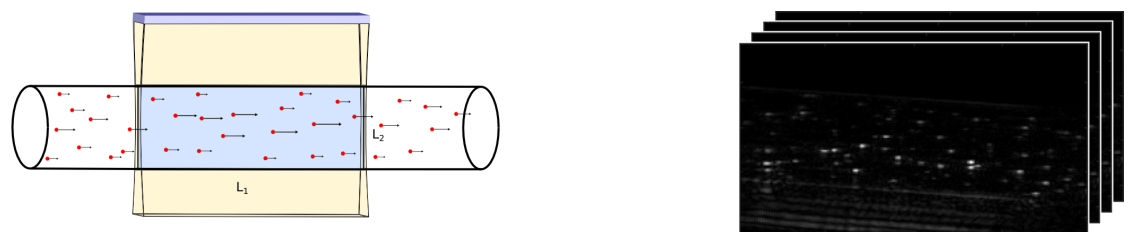

Figure 1.1: A schematic representation of the plane wave ultrasound Echo PIV setup (left). In a rigid cylindrical tube of inner radius $R=L_{2} / 2$ a liquid flows that is seeded with bubbles, typically microbubbles. A linear transducer array is placed along the tube axis and transmits a plane wave acoustic pulse into inhomogeneous field. Then the same transducer records the backscattered acoustic wave that is reflected and scattered by static tube walls and dynamic bubbles. The transmission and recording step is repeated very fast (faster than $1000 \mathrm{~Hz}$ ). This produces an image sequence of high temporal resolution (right) that displays speckle patterns driven by the flow.

of Echo PIV to blood flow estimation. Here, we restrict our attention to estimating the peak velocity of pipe flows. The velocity field in a laminar and steady flow (a.k.a. Poiseuille flow) is given by

$$
u=u(x)=\left(u_{1}\left(x_{2}\right), 0\right)^{\top}, \quad u_{1}\left(x_{2}\right)=v_{m}\left(1-\left(\frac{x_{2}}{R}\right)^{2}\right), \quad v_{m} \geq 0,
$$

where $v_{m}$ denotes the peak velocity in a pipe of size $R$ assumed to be centered at $x_{2}=0$. Thus, the flow has a parabolic profile, does not depend on $x_{1}$, and hence has a single degree of freedom $v_{m}$. See Figure 1.2, left panel, for an illustration. The flow cannot be directly measured but must be estimated. We achieve this by estimating the parameter $v_{m}$ directly using the data of a given image sequence, as shown in the right panel of Figure 1.1.

A common assumption of experimental fluid dynamics [RWWK07] is that the flow has been seeded with a set of randomly located particles (Figure 1.2, right panel), also called tracer particles, that follow the flow dynamics. Motion is estimated via the displacement of these tracer particles. Yet, the individual particles cannot be directly tracked in the flow. Rather, what can be directly observed is a moving 'texture' of speckle patterns driven by the flow. In this work, we propose an approach for estimating the flow directly from image sequence data, based on a model of the image sequence $f$ in terms of tracer particles with unknown locations $\left\{x^{(i)}\right\}_{i \in N_{p}}, x^{(i)}=\left(x_{1}^{(i)}, x_{2}^{(i)}\right)^{\top}$ and corresponding time independent unknown velocities $u^{(i)}=\left(u_{1}^{(i)}, 0\right)^{\top}$, where $u^{(i)}=u\left(x^{(i)}\right)$ are given by (1.1). By utilizing the model of $f$ and its Fourier transform, we obtain a simple global approach for robustly estimating the peak velocity $v_{m}$ directly from given noisy image sequence data. 

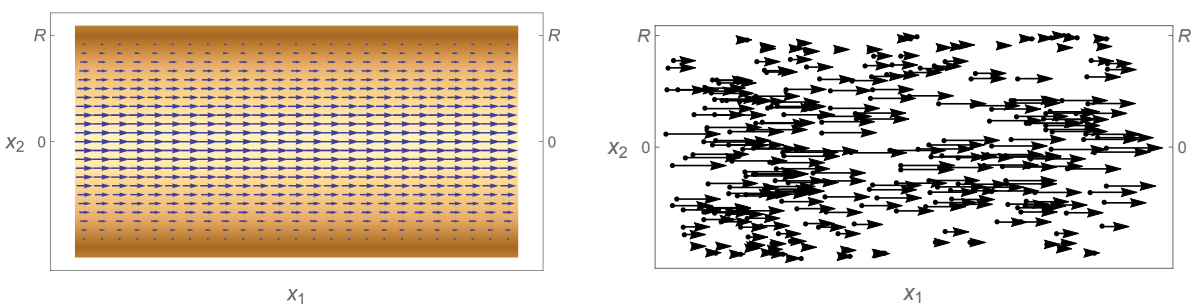

Figure 1.2: LEFT: A Poiseuille flow governed by (1.1). The background illustrates the parabolic velocity profile parametrized by the pipe size $R$ and the peak velocity $v_{m}$. RIGHT: An unknown set of randomly located particles that are moving with the flow is the starting point of our model of the imaging process that generates an image sequence of a moving texture (cf. Section 1). The task is to robustly estimate the unknown peak velocity $v_{m}$ under adverse imaging conditions from noisy image sequence data.

\subsection{Related Work and Contribution}

Research on plane wave Echo PIV is concerned with image reconstruction and motion estimation. In this work, we only focus on the motion estimation and point out that image reconstruction is based on the inverse scattering model presented in [BSPS16]. We propose a novel global approach to estimate the maximum flow velocity $v_{m}$ by exploiting geometry of the spectrum of the image sequence signal in the spatio-temporal Fourier domain. Compared to dictionary-based velocimetry [BPPS16, BGPS15], the proposed method handles the noise better and reduces the computational cost.

Our model exploits the basic fact that any motion can be locally approximated by a translation, together with the representation of translated functions by the Fourier transform. This viewpoint has a long tradition in image processing and computer vision [Hee88, FJ90]. Our approach to image sequence data recorded by Echo PIV is novel.

\subsection{Organization}

Our approach is described in Section 2 and Section 3. The spectrum of the input image sequence in characterized in Section 2, while Section 3 details the peak velocity estimation approach based on the support of this spectrum. In Section 4 we validate our approach experimentally. 


\section{Poiseuille Image Sequence Spectra}

We adopt the particle scenario of Figure 1.2, right panel, and a spectral representation of the motion of all particles driven by an unknown Poiseuille flow. This provides a basis for global motion estimation in order to better cope with the fact that no particle locations are known nor can individual particles be directly observed.

The $d$-dimensional Fourier transform and its inverse are given by

$$
\begin{gathered}
\hat{f}(\omega)=\mathcal{F}(f)(\omega)=\int_{\mathbb{R}^{d}} f(x) e^{-i\langle\omega, x\rangle} \mathrm{d} x, \\
f(x)=\mathcal{F}^{-1}(\hat{f})(x)=\frac{1}{(2 \pi)^{d}} \int_{\mathbb{R}^{d}} \hat{f}(w) e^{+i\langle\omega, x\rangle} \mathrm{d} \omega,
\end{gathered}
$$

where $\langle\omega, x\rangle=\sum_{i \in[d]} \omega_{i} x_{i}$ denotes the Euclidean inner product and $[d]:=\{1,2, \ldots, d\}, d \in \mathbb{N}$.

The Fourier transform is a one-to-one mapping on the Schwartz space $\mathcal{S}\left(\mathbb{R}^{d}\right)$ of functions with rapidly decreasing derivatives of any order. It can be extended by duality to the space $\mathcal{S}^{\prime}\left(\mathbb{R}^{d}\right)$ of tempered distributions, i.e. the space of linear and continuous functionals acting on $\mathcal{S}\left(\mathbb{R}^{d}\right)$. We refer to e.g. [Geo15, Ch. 8.1-3] for details.

Remark 2.1. It will be convenient and more informative for readers from various fields to simply speak of 'functions' in this paper even if a distribution actually is meant. For example, the image sequence function $f(x, t)$ given by (2.4) actually is an element of $\mathcal{S}^{\prime}\left(\mathbb{R}^{3}\right)$.

The Delta function (Dirac distribution) supported at $x_{0} \in \mathbb{R}^{d}$ is denoted by $\delta(x-$ $x_{0}$ ). The Fourier transform of the Delta function (in the sense of distributions) is given by

$$
\hat{\delta}(\omega)=e^{-\langle\omega, 0\rangle} \equiv 1 .
$$

The Fourier transform of a function $T_{x_{0}} f(x):=f\left(x-x_{0}\right)$ translated by $x_{0} \in \mathbb{R}^{d}$ is

$\mathcal{F}\left(T_{x_{0}} f\right)(\omega)=\int_{\mathbb{R}^{d}} f\left(x-x_{0}\right) e^{-i\langle\omega, x\rangle} \mathrm{d} x=\int_{\mathbb{R}^{d}} f(z) e^{-i\left\langle\omega, z+x_{0}\right\rangle} \mathrm{d} z=\hat{f}(\omega) e^{-i\left\langle\omega, x_{0}\right\rangle}$.

We describe a set of $N_{p}$ non-interacting particles that move with the flow by the function*

$$
f(x, t)=\sum_{i \in\left[N_{p}\right]} \delta\left(x-x^{(i)}-u^{(i)} t\right), \quad u^{(i)}=u\left(x^{(i)}\right),
$$

\footnotetext{
* Recall the convention due to Remark 2.1 .
} 
where $u^{(i)}=\left(u_{1}^{(i)}, 0\right)^{\top}$ and $x^{(i)}=\left(x_{1}^{(i)}, x_{2}^{(i)}\right)^{\top}$ are the time independent velocity and initial position of particle $i$, respectively, and $u^{(i)}$ is given by (1.1). Both the filter design and the approach for estimating the flow will be based on the Fourier transform of the abstract model (2.4) of the image sequence $f$.

Proposition 2.1. Let $\omega=\left(\omega_{x}^{\top}, \omega_{3}\right)^{\top}=\left(\omega_{1}, \omega_{2}, w_{3}\right)^{\top}$ denote the angular frequency vector. Then the Fourier transform of the image sequence function (2.4) is given by

$$
\hat{f}(\omega)=\hat{f}\left(\omega_{x}, \omega_{3}\right)=\sum_{i \in\left[N_{p}\right]} e^{-i\left\langle\omega_{x}, x^{(i)}\right\rangle} \delta\left(\omega_{1} u_{1}^{(i)}+\omega_{3}\right) .
$$

Proof. Let $h(x)=h\left(x_{1}, x_{2}\right)$ denote an arbitrary 2D image function. Then, for any fixed vector $u \in \mathbb{R}^{2}$, the image sequence function $\tilde{h}(x, t)=h(x-u t)$ corresponds to the translation of the function $h(x)$ with constant velocity $u$. Applying the 3D Fourier transform to this image sequence yields

$$
\begin{aligned}
\mathcal{F}(\tilde{h}) & =\int_{\mathbb{R}^{3}} h(x-u t) e^{-i\left(\left\langle\omega_{x}, x\right\rangle+w_{3} t\right)} \mathrm{d} x \mathrm{~d} t=\int_{\mathbb{R}^{2}} h(z) e^{-i\left\langle\omega_{x}, z\right\rangle} \mathrm{d} z \int_{\mathbb{R}} e^{-i\left(\left\langle\omega_{x}, u\right\rangle+\omega_{3}\right) t} \mathrm{~d} t \\
& =\hat{h}\left(\omega_{x}\right) \delta\left(\left\langle\omega_{x}, u\right\rangle+\omega_{3}\right),
\end{aligned}
$$

where the evaluation of the last integral follows from (2.2) and (2.3). Now, setting $h^{(i)}(x)=\delta\left(x-x^{(i)}\right)$, Eq. (2.4) reads

$$
f(x, t)=\sum_{i \in N_{p}} h^{(i)}\left(x-u^{(i)} t\right) .
$$

Applying relation (2.6) and taking into account the linearity of the Fourier transform, we get

$$
\hat{f}(\omega)=\sum_{i \in N_{p}} \hat{h}^{(i)}\left(\omega_{x}\right) \delta\left(\left\langle\omega_{x}, u^{(i)}\right\rangle+\omega_{3}\right) \stackrel{(2.3)}{=} \sum_{i \in N_{p}} e^{-i\left\langle\omega_{x}, x^{(i)}\right\rangle} \delta\left(\left\langle\omega_{x}, u^{(i)}\right\rangle+\omega_{3}\right),
$$

which due to the specific form (1.1) of the flow, is equal to (2.5).

Equation (2.5) says that the spectrum $\hat{f}$ of the image sequence $f$ is the sum of complex phase functions on a corresponding pencil of planes through the origin $\omega=$ 0 with normal vectors $n^{(i)}=\left(u_{1}^{(i)}, 0,1\right)^{\top}$. Figure 2.1 depicts the resulting support of $\hat{f}(\omega)$, which is bounded by two extremal planes corresponding to zero velocity $u=0$ and normal $\left(0,0, \omega_{3}\right)^{T}$, and to the peak velocity $u=\left(v_{m}, 0\right)^{\top}$ and normal $\left(v_{m}, 0,1\right)^{\top}$, respectively.

This finding suggests to estimate the peak velocity $v_{m}$ and hence the full flow due to (1.1) by estimating the spectral support of the Fourier transform of a given image sequence. We describe our approach in the next section. 


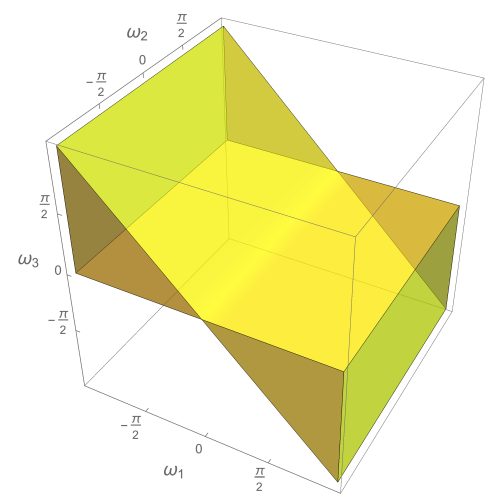

Figure 2.1: Spectral support of the Fourier transform of an image sequence corresponding to the Poiseuille pipe flow model. This non-pointed cone, that extends along the $\omega_{2}$ axis, is bounded by two planes including the plane $\omega_{3}=0$. The normal vector of the other plane depends on the unknown peak velocity $v_{m}$ that we wish to estimate from image sequence data.

\section{Peak Velocity Estimation}

We derive in Section 3.1 a piecewise linear model of the cone geometry depicted in Figure 2.1, that takes into account noise suppression and the symmetry of real signals in the complex Fourier domain. Based on this model, a numerical method for robustly estimating the spectral support of a real image sequence is developed in Section 3.2.

\subsection{Direct Spectral Support Estimation}

The cone shown in Figure 2.1 is bounded by the box $[-\pi, \pi] \times[-\pi, \pi] \times[-\pi, \pi]$ and the planes $\omega_{3}=0, v_{m} \omega_{1}+\omega_{3}=0$. Due to the high noise level of real data, we discard the spectrum at large frequencies as well as a redundant half-space due to the symmetry of real signals $f(x, t)$ in Fourier space. As a consequence, we only consider the spectrum in the smaller box $[0, \pi / 4] \times[-\pi / 4, \pi / 4] \times[-\pi / 4,0]$.

Assuming a uniform distribution of the amplitude spectrum

$$
|\hat{f}(\omega)| \approx C_{f}, \quad C_{f}>0
$$

of the image sequence signal $f(x, t)$, for some constant $C_{f}>0$, which is justified by (2.4) $\left(N_{p}\right.$ is unknown) and (2.2), we define the region

$$
\Omega(v)=\left\{\omega \in \mathbb{R}^{3}: 0 \leq \omega_{1} \leq \frac{\pi}{4},-\frac{\pi}{4} \leq \omega_{2} \leq \frac{\pi}{4},-\min \left(v \omega_{1}, \frac{\pi}{4}\right) \leq \omega_{3} \leq 0\right\}, v \geq 0
$$




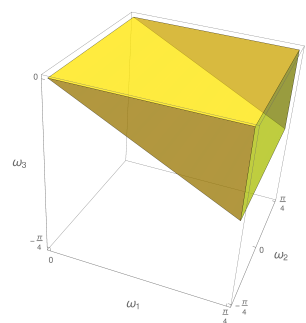

(a)

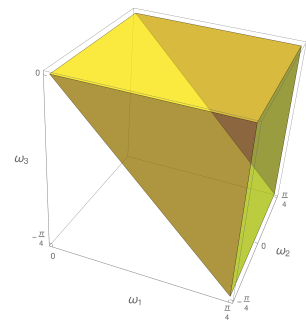

(b)

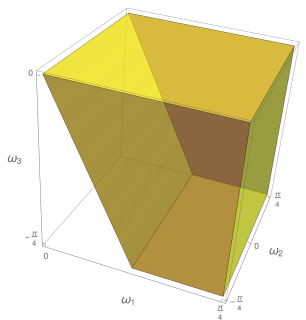

(c)

Figure 3.1: This figure illustrates the set $\Omega\left(v_{m}\right)$ (a) for $v_{m}<1$, (b) for $v_{m}=1$, and (c) for $v_{m}>1$, that defines the relevant spectral support of $\hat{f}$. Conversely, estimating the actual support for a given spectrum $\hat{f}$ corresponding to real image sequence data $f$, enables to estimate the parameter $v_{m}$.

and estimate the spectral support of $\hat{f}$ by the volume integral

$$
s(v)=\frac{1}{\left|\Omega\left(v_{m}\right)\right|} \int_{\Omega(v)}|\hat{f}(\omega)| \mathrm{d} \omega, \quad v_{m}, v>0
$$

which in practice, for $\hat{f}$ corresponding to real data, is computed using the FFT and evaluating numerically a Riemannian sum that accurately approximates (3.3). Note, that the support of $|\hat{f}(\omega)|$ is restricted to $\Omega\left(v_{m}\right)$, and that the volume integral attains its maximum when $v \geq v_{m}$, in which case $s(v)=C_{f}$.

To obtain an analytical model of $s(v)$ given by (3.3), we have to distinguish between two cases.

Case 1: If $v_{m} \leq 1$, then $\left|\Omega\left(v_{m}\right)\right|=\left(\frac{\pi}{4}\right)^{3} v_{m}$ and (3.3) has the form of a piecewise linear function

$$
s(v)= \begin{cases}\frac{C_{f}}{v_{m}} v, & 0 \leq v<v_{m} \\ C_{f}, & v \geq v_{m}\end{cases}
$$

This case is illustrated in Figure 3.2 (a) and (b).

Case 2: If $v_{m}>1$, then $\left|\Omega\left(v_{m}\right)\right|=\left(\frac{\pi}{4}\right)^{3}\left(2-\frac{1}{v_{m}}\right)$ and (3.3) is given by

$$
s(v)= \begin{cases}\frac{C_{f}}{2-\frac{1}{v_{m}}} v, & 0 \leq v<1, \\ \frac{C_{f}}{2-\frac{1}{v_{m}}}\left(1-\frac{1}{v}\right), & 1 \leq v<v_{m}, \\ C_{f}, & v \geq v_{m} .\end{cases}
$$


This case is illustrated in Figure 3.2 (c).
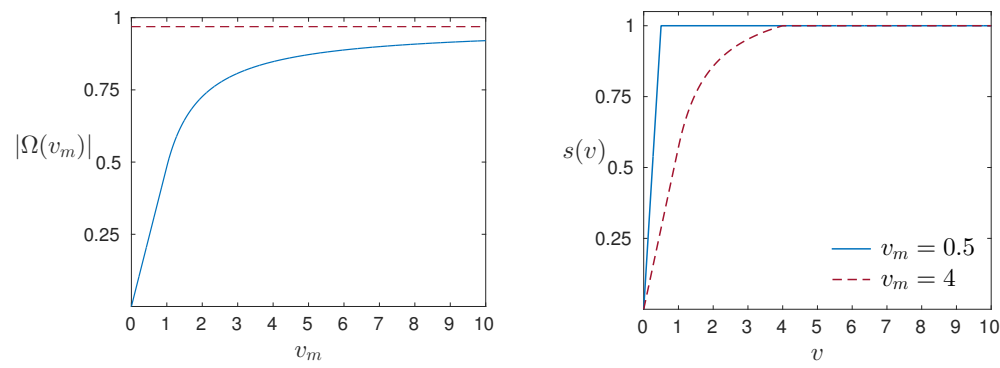

Figure 3.2: (Left) The dependence $\left|\Omega\left(v_{m}\right)\right|$ on $v_{m}$. The red dashed line is the upper bound of $\left|\Omega\left(v_{m}\right)\right|$ as $v_{m} \rightarrow \infty$ that equals $2\left(\frac{\pi}{4}\right)^{3}$. (Right) The normalized function $s(v)$ for $v_{m}<1$ (case 1 ) and $v_{m}>1$ (case 2).

\subsection{Parameter Estimation by Robust Numerical Piecewise Fitting}

We first develop smooth parametric representations of (3.4) and (3.5), respectively, that are amenable to efficient numerical optimization, followed by sketching the numerical approach.

\subsubsection{Case $v_{m} \leq 1$.}

Equation (3.4) suggests that the data set $\left\{\hat{v}_{i}, s\left(\hat{v}_{i}\right)\right\}_{i=1, \ldots, n}$ estimated numerically from (3.3) via the Riemannian sum is best approximated by a two-segment piecewise linear continuous function of the form

$$
g: \mathbb{R} \mapsto \mathbb{R}, \quad g(x)= \begin{cases}a_{1}+b_{1} x, & x \leq \xi, \\ a_{2}+b_{2} x, & x>\xi .\end{cases}
$$

We define

$$
l_{1}(x)=a_{1}+b_{1} x, \quad l_{2}(x)=a_{2}+b_{2} x,
$$

and rewrite (3.6) as

$$
g(x)=-\max \left\{-l_{1}(x),-l_{2}(x)\right\} .
$$

A smooth approximation of this concave function can be achieved by using the logexponential function,

$$
g_{\varepsilon}(x)=-\varepsilon \ln \left(\mathrm{e}^{-l_{1}(x) / \varepsilon}+\mathrm{e}^{-l_{2}(x) / \varepsilon}\right),
$$


where $\varepsilon>0$ is the smoothing parameter that enables a uniform approximation of (3.8) as $\varepsilon \rightarrow 0$ [RW09, Ch. 1.-H].

Using (3.7) and the continuity of $g(x)$ at the breakpoint $\xi$ which implies that $a_{1}+b_{1} \xi=a_{2}+b_{2} \xi$, we express (3.9) as

$$
\begin{aligned}
g_{\varepsilon}(x) & =-\varepsilon \ln \left(\mathrm{e}^{-\left(a_{1}+b_{1} x\right) / \varepsilon}+\mathrm{e}^{-\left(a_{1}+\left(b_{1}-b_{2}\right) \xi+b_{2} x\right) / \varepsilon}\right) \\
& =\alpha+\beta x-\varepsilon \ln \left(1+\mathrm{e}^{-\gamma(x-\xi) / \varepsilon}\right),
\end{aligned}
$$

where

$$
\alpha=a_{1}, \quad \beta=b_{1}, \quad \text { and } \quad \gamma=b_{2}-b_{1} .
$$

3.2.2 Case $v_{m}>1$.

In view of (3.5) we consider the piecewise non-linear continuous function

$$
h(x): \mathbb{R}_{+} \backslash\{0\} \mapsto \mathbb{R}, \quad h(x)= \begin{cases}a_{1}+b_{1} x, & 0<x \leq \xi_{1}, \\ a_{2}+\frac{b_{2}}{x}, & \xi_{1}<x \leq \xi_{2}, \\ a_{3}+b_{3} x, & x<\xi_{2},\end{cases}
$$

where $0<\xi_{1} \leq \xi_{2}$ are the breakpoints.

Lemma 3.1. For two breakpoints $0<\xi_{1} \leq \xi_{2}$ the function defined in (3.12) has the canonical representation

$$
h(x)=\alpha+\beta x+\sum_{i=\{1,2\}} \delta_{i}\left|\frac{1}{x}-\frac{1}{\xi_{i}}\right|+\sum_{i=\{1,2\}} \phi_{i}\left|x-\xi_{i}\right|
$$

where

$$
\alpha=\frac{a_{1}+a_{3}}{2}, \beta=\frac{b_{1}+b_{3}}{2}, \delta_{1}=-\delta_{2}=-\frac{b_{2}}{2}, \phi_{1}=-\frac{b_{1}}{2}, \text { and } \phi_{2}=\frac{b_{3}}{2} .
$$

Proof. The result follows immediately from the continuity of $h(x)$ at the breakpoints $\xi_{1}, \xi_{2}$ and the expansion of absolute value terms for the cases $0<x \leq \xi_{1}, \xi_{1}<x \leq$ $\xi_{2}$ and $x>\xi_{2}$.

Lemma 3.2. The piecewise non-linear function (3.13) can be approximated uniformly by the smooth function

$h_{\varepsilon}(x)=A+B x+\sum_{i=\{1,2\}} D_{i} \ln \left(1+\mathrm{e}^{-2\left(\frac{1}{x}-\frac{1}{\xi_{i}}\right) / \varepsilon}\right)+\sum_{i=\{1,2\}} F_{i} \ln \left(1+\mathrm{e}^{-2\left(x-\xi_{i}\right) / \varepsilon}\right)$,

where $A=\alpha-\delta_{1} / \xi_{1}-\delta_{2} / \xi_{2}-\phi_{1} \xi_{1}-\phi_{2} \xi_{2}, B=\beta+\phi_{1}+\phi_{2}, D_{i}=\varepsilon \delta_{i}$ and $F_{i}=\varepsilon \phi_{i}$. 
Proof. The absolute value of $x \in \mathbb{R}$ can be expressed as

$$
|x|=\max \{-x, x\},
$$

and, similar as discussed above, is approximated uniformly by the smooth function

$$
|x|_{\varepsilon}=\varepsilon \ln \left(\mathrm{e}^{-x / \varepsilon}+\mathrm{e}^{x / \varepsilon}\right)=x+\varepsilon \ln \left(1+\mathrm{e}^{-2 x / \varepsilon}\right) .
$$

The replacement of the absolute value terms in (3.13) with the corresponding smooth approximations $\left|\frac{1}{x}-\frac{1}{\xi_{i}}\right|_{\varepsilon}$ and $\left|x-\xi_{i}\right|_{\varepsilon}$, for $i=1,2$, yields (3.15).

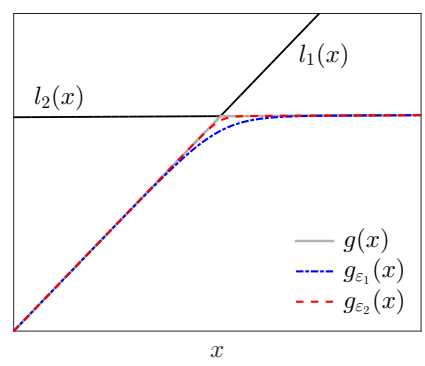

(a)

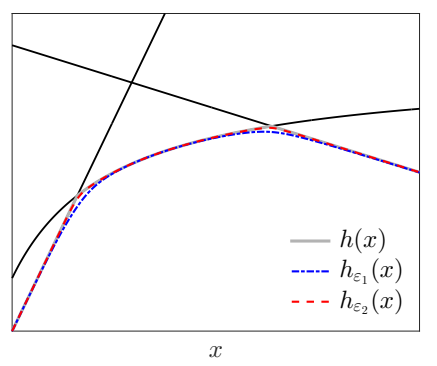

(b)

Figure 3.3: Smooth approximation of two-segment piecewise linear functions defined in (3.6) and of the piecewise non-linear function defined in (3.12), for smoothing parameter values $\varepsilon_{1}=0.1$ and $\varepsilon_{2}=0.03$.

\subsubsection{Numerical Optimization}

Returning to our application, equations (3.4) and (3.5) suggest that the breakpoints $\xi$ and $\xi_{2}$ in (3.6) and (3.12), respectively, correspond to the unknown flow parameter $v_{m}>0$. We define for some small $\varepsilon>0$ the functions

$$
\begin{gathered}
\hat{g}_{\varepsilon}: \mathbb{R}^{n} \times \mathbb{R}^{4} \rightarrow \mathbb{R}^{n}, \quad \hat{g}_{\varepsilon}(\hat{v} ; \alpha, \beta, \gamma, \xi)=\alpha \mathbb{1}+\beta \hat{v}-\varepsilon \ln \left(\mathbb{1}+\mathrm{e}^{-\gamma(\hat{v}-\xi \mathbb{1}) / \varepsilon}\right) \\
\hat{h}_{\varepsilon}: \mathbb{R}_{+}^{n} \backslash\{0\} \times \mathbb{R}^{5} \times \mathbb{R}_{+} \backslash\{0\} \rightarrow \mathbb{R}^{n} \\
\hat{h}_{\varepsilon}\left(\hat{v} ; \alpha, \beta, \delta, \phi_{1}, \phi_{2}, \xi_{2}\right)=\left(\alpha-\delta / \xi_{1}+\delta / \xi_{2}-\phi_{1} \xi_{1}-\phi_{2} \xi_{2}\right) \mathbb{1}+ \\
\left(\beta+\phi_{1}+\phi_{2}\right) \hat{v}+\varepsilon \ln \frac{\left(\mathbb{1}+\mathrm{e}^{-2\left(\frac{1}{\hat{v}}-\frac{1}{\xi_{1}} \mathbb{1}\right) / \varepsilon}\right)^{\delta}\left(\mathbb{1}+\mathrm{e}^{-2\left(\hat{v}-\xi_{1} \mathbb{1}\right) / \varepsilon}\right)^{\phi_{1}}}{\left(\mathbb{1}+\mathrm{e}^{-2\left(\frac{1}{\hat{v}}-\frac{1}{\xi_{2}} \mathbb{1}\right) / \varepsilon}\right)^{\delta}\left(\mathbb{1}+\mathrm{e}^{-2\left(\hat{v}-\xi_{2} \mathbb{1}\right) / \varepsilon}\right)^{-\phi_{2}}}
\end{gathered}
$$


where $\delta=\delta_{1}=-\delta_{2}$ and $\xi_{1}=1$ is fixed. In both definitions, subdivision, the logarithmic and exponential functions are applied component-wise. Using the nonmonotone spectral projected gradient method [BMR00] we minimize numerically the functions

$$
\begin{gathered}
f_{g}(\alpha, \beta, \gamma, \xi)=\frac{1}{2}\left\|\hat{g}_{\varepsilon}(\hat{v} ; \alpha, \beta, \gamma, \xi)-\hat{s}(\hat{v})\right\|_{2}^{2}, \quad 0 \leq \xi \leq 1, \\
f_{h}\left(\alpha, \beta, \delta, \phi_{1}, \phi_{2}, \xi_{2}\right)=\frac{1}{2}\left\|\hat{h}_{\varepsilon}\left(\hat{v} ; \alpha, \beta, \delta, \phi_{1}, \phi_{2}, \xi_{2}\right)-\hat{s}(\hat{v})\right\|_{2}^{2}, \quad \xi_{2} \geq 1 .
\end{gathered}
$$

where $\hat{v}=\left(\hat{v}_{1}, \ldots, \hat{v}_{n}\right)^{T} \in \mathbb{R}^{n}$ and $\hat{s}(\hat{v}) \in \mathbb{R}^{n}$, with respect to all parameters. The functions $\hat{g}_{\varepsilon}$ and $\hat{h}_{\varepsilon}$ are smooth with respect $\alpha, \beta, \gamma, \xi$ and $\alpha, \beta, \delta, \phi_{1}, \phi_{2}, \xi_{2}$, respectively, which implies the smoothness of $f_{g}$ and $f_{h}$. The constraints on variables $\xi$ and $\xi_{2}$ express the model conditions on the breakpoints for each case specified in (3.4) and (3.5).

The resulting numerically computed optimal values of $\xi$ and $\xi_{2}$ yield an estimate of the unknown maximal velocity $v_{m}$. We illustrate this approach in the next section by estimating $v_{m}$ with proposed method on synthetic and real data.

\section{Experiments}

We demonstrate the proposed method for estimating the maximal velocity $v_{m}$ using both synthetic and real ultrasound data. The synthetic scenes are generated in order to validate the method based on ground truth, and to show that the method copes with a broad range of realistic 'moving textures' that cover those occuring in practice. The real data experiments demonstrate that our method is robust against noise.

Numerical experiments were conducted using MATLAB R2015a on a $2.7 \mathrm{GHz}$ Intel Core i7-7500U processor with $16 \mathrm{~GB}$ of RAM memory and running of GNU/Linux operating system (elementary OS 0.4 64Bit). For the optimization problem we used the SPG1 version of the non-monotone projected gradient algorithm presented in [BMR00]. All the parameters related to the initialization of the algorithm were set to the values given in the original paper. For the non-monotone parameter we decided to use $M=50$. We stopped the execution of the algorithm after 500 and 3000 iterations for the piecewise linear model and piecewise non-linear model, respectively. The running time for FFT, etimation of $\hat{s}(\hat{v})$, and optimization was between $2-10$ seconds depending on the data sets and number of iterations.

\subsection{Synthetic Data}

We analysed four synthetic textures, see Figure 4.1, that moved according to Poiseuille flow model (1.1). The considered textures are: 


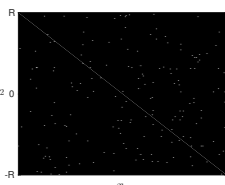

(a)

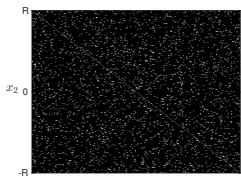

(b)

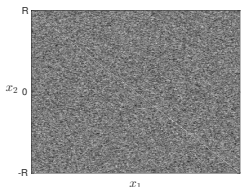

(c)

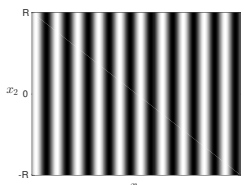

(d)

Figure 4.1: Considered textures: (a) uniform distribution of particles at low density, (b) uniform distribution of particles at high density, (c) uniform white noise, and (d) two dimensional sinusoid.

(a) uniform distribution of Dirac particles at low density,

(b) uniform distribution of Dirac particles at high density,

(c) white noise, and

(d) two dimensional sinusoid.

Figure 4.2 depicts the deformation of the sinusoid under the parabolic velocity field.

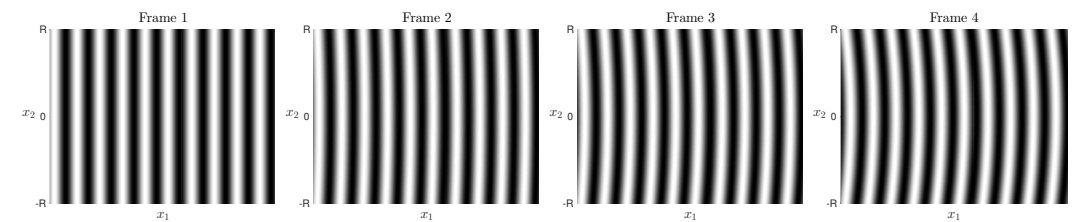

Figure 4.2: The first four frames show the Poiseuille flow of a two dimensional sinusoid.

All image sequences consist of 256 temporal frames of size $256 \times 256$ pixels. The flow direction was from left to right with periodic boundary conditions imposed at the right boundary. The Fourier spectrum for each sequence was computed with the fast Fourier transform (FFT). We considered two cases: $v_{m} \leq 1$ and $v_{m}>1$.

\subsubsection{Case $v_{m} \leq 1$.}

We generated a Poiseuille flow with peak velocity $v_{m}^{*}=0.5$ pixels $/$ frame. The results are shown in Figure 4.3. The numerically estimated parameter values are listed in Table 1 . Due to the small subpixel peak velocity $v_{m}=0.5$ pixel/frame which, due to the parabolic profile (cf. Fig. 1.2) is noticeable only in the center region of the pipe, we obtained a slight but systematic overestimation of the peak velocity. 

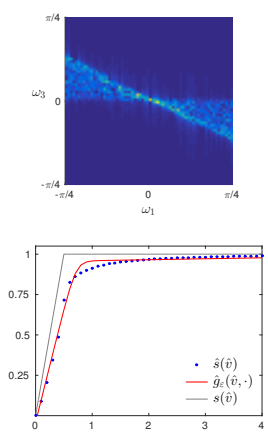

(a)
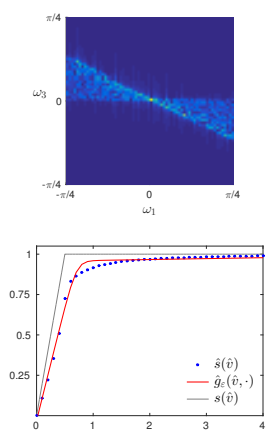

(b)

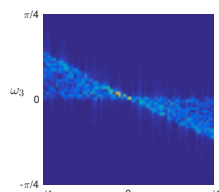

0
$\omega_{1}$

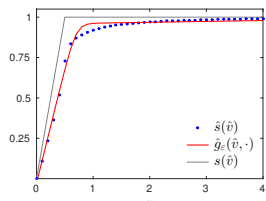

(c)
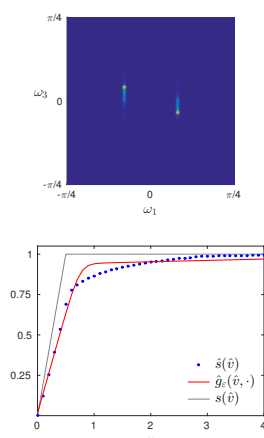

(d)

Figure 4.3: First row. Fourier spectrum $|\hat{f}(\omega)|$ of the image sequence signal for each texture moving with the maximum flow velocity $v_{m}=0.5$ pixels/frame. The images illustrate a section of the spectral support by the plane $\left(\omega_{1}, 0, \omega_{3}\right)$ in the frequency domain. Second row. Numerical evaluation of the integral (3.3) is shown by the blue dotted line. The red solid line shows the corresponding fit of the piecewise linear function (3.10b), with $\varepsilon=0.1$, that minimizes (3.22). The gray line shows the normalized $s(v)$ calculated from (3.4) for $v_{m}^{*}=0.5$. The numerically estimated parameter values are listed in Table 1.

\begin{tabular}{|l|c|c|c|c|}
\hline & $\alpha$ & $\beta$ & $\gamma$ & $\xi=v_{m}$ \\
\hline Reference & 0 & 2.00 & -2.00 & 0.500 \\
\hline low density & -0.041 & 1.403 & -1.39 & 0.713 \\
\hline high density & -0.031 & 1.408 & -1.402 & 0.703 \\
\hline white noise & -0.028 & 1.418 & -1.412 & 0.698 \\
\hline sinusoid & 0.015 & 1.247 & -1.239 & 0.744 \\
\hline
\end{tabular}

Table 1: Parameter values estimated by minimizing (3.22). The reference row gives the theoretical parameters values defined by (3.11) for $v_{m}^{*}=0.5$, based on the two segment model (3.4) and (3.6). Our approach slightly overestimates the small subpixel peak velocity.

\subsubsection{Case $v_{m}>1$.}

We generated a Poiseuille flow with peak velocity $v_{m}^{*}=5$ pixels/frame. The results are shown in Figure 4.4 and discussed in the caption. The numerically estimated parameter values are listed in Table 2 . Since the peak velocity is much larger than the subpixel velocity considered in the previous section, we obtained fairly accurate 
estimates of $v_{m}$, except for the sinusoid sequence which is extremely unrealistic and deviates from our image sequence model (2.4).
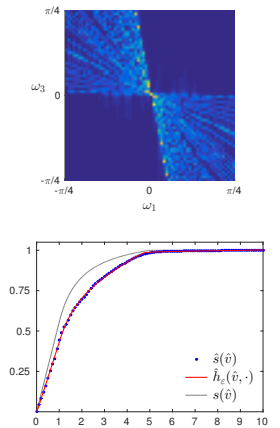

(a)
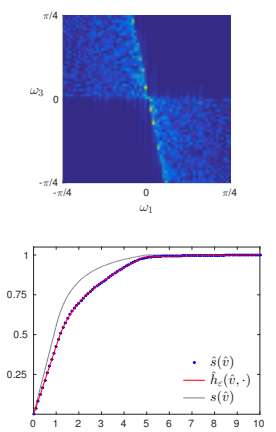

(b)
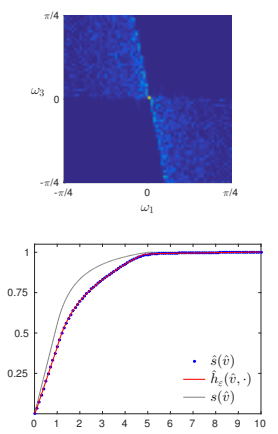

(c)

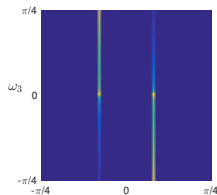

$\omega_{1}$

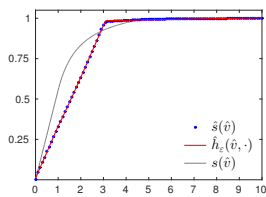

(d)

Figure 4.4: First row. Fourier spectrum $|\hat{f}(\omega)|$ of the image sequence signal for each texture moving with the maximum flow velocity $v_{m}=5$ pixels/frame. The images illustrate a section of the spectral support by the plane $\left(\omega_{1}, 0, \omega_{3}\right)$ in the frequency domain. Second row. The numerical evaluation of the integral (3.3) for several $v$ values that constitute the data set $(\hat{v}, \hat{s}(\hat{v}))$ is depicted by the blue dotted line. The red solid line shows the smooth piecewise non-linear function defined by (3.15), with $\varepsilon=0.03$, that minimizes (3.23). The gray line shows the normalized $s(v)$ calculated from (3.5) for $v_{m}=5$. The numerically estimated parameter values are listed in Table 2.

\begin{tabular}{|l|c|c|c|c|c|c|}
\hline & $\alpha$ & $\beta$ & $\delta$ & $\phi_{1}$ & $\phi_{2}$ & $\xi_{2}=v_{m}$ \\
\hline Reference & 0.500 & 0.273 & 0.273 & -0.273 & 0 & 5.000 \\
\hline low density & 0.486 & 0.220 & 0.214 & -0.189 & -0.028 & 4.754 \\
\hline high density & 0.489 & 0.216 & 0.215 & -0.187 & -0.027 & 4.880 \\
\hline white noise & 0.487 & 0.211 & 0.230 & -0.181 & -0.028 & 4.797 \\
\hline sinusoid & 0.493 & 0.164 & -0.029 & 0.003 & -0.164 & 3.086 \\
\hline
\end{tabular}

Table 2: Parameter values estimated by minimizing (3.23). The reference row gives the theoretical parameters values defined by (3.14) for $v_{m}^{*}=5$, based on the piecewise non-linear function (3.5) and (3.12). Except for the sinusoid sequence that deviates from our image sequence model (2.4), all parameter estimates are fairly accurate. 


\begin{tabular}{|l|c|c|}
\hline & air-bubbles flow & microbubbles flow \\
\hline Tube radius, $R$ & $0.005 \mathrm{~m}$ & $0.005 \mathrm{~m}$ \\
\hline Temporal frames & 299 & 99 \\
\hline Image size, $N_{1} \times N_{2}$ & $372 \times 135$ & $396 \times 131$ \\
\hline Frame rate & $6.666 \mathrm{kHz}$ & $2.222 \mathrm{kHz}$ \\
\hline Temporal resolution, $\Delta t$ & $0.150 \mathrm{~ms}$ & $0.450 \mathrm{~ms}$ \\
\hline Field of view size, $L_{1} \times L_{2}$ & $3.8 \times 1 \mathrm{~cm}^{2}$ & $3.8 \times 1 \mathrm{~cm}^{2}$ \\
\hline Volume flow rate, $\Delta \Phi$ & $15 \mathrm{ml} / \mathrm{s}$ & $40 \mathrm{ml} / \mathrm{s}$ \\
\hline Estimated $v_{m}^{*}$, in $\mathrm{m} / \mathrm{s}$ & 0.382 & 1.019 \\
\hline Estimated $v_{m}^{*}$, in $\mathrm{px} /$ frame & 0.561 & 4.777 \\
\hline
\end{tabular}

Table 3: Real plane wave ultrasound experiments: relevant parameters and reference values.

\subsection{Real Data}

We evaluated the proposed method on two sets of real plane wave ultrasound images for bubbles flow in a straight tube. The in vitro experiment was performed under controlled conditions and the volume flow rate was measured using a flow-meter. Table 3 summarizes the relevant imaging parameters. We refer to $\left[\mathrm{VKV}^{+} 16\right]$ for more details about the experiment.

Under the assumption that the captured flow is fully developed, laminar and steady, we estimate the peak velocity from the measured volume flow rate $\Delta \Phi$ given by

$$
v_{m}^{*}=2 \frac{\Delta \Phi}{\pi R^{2}} \cdot \frac{N_{1} \Delta t}{L_{1}}
$$

where $R$ is the radius of the tube and the last term converts the velocity units form $\mathrm{m} / \mathrm{s}$ into pixels/frame. The numerical results for the estimated peak velocity are given in Table 3, and we used them as reference values.

\begin{tabular}{|c|c|c|c|c|c|}
\hline & $\alpha$ & $\beta$ & $\gamma$ & $\xi=v_{m}$ & $v_{m}^{*}$ \\
\hline air-bubbles flow & -0.0219 & 1.166 & -1.162 & 0.828 & 0.561 \\
\hline
\end{tabular}

Table 4: Parameters and peak velocity estimate for the air-bubbles flow shown in Figure 4.5. Similar to the synthetic sequences with a small subpixel peak velocity (cf. Fig. 4.3 and Table 1), we obtained a slight overestimate relative to the independent measurement $v_{m}^{*}$. 

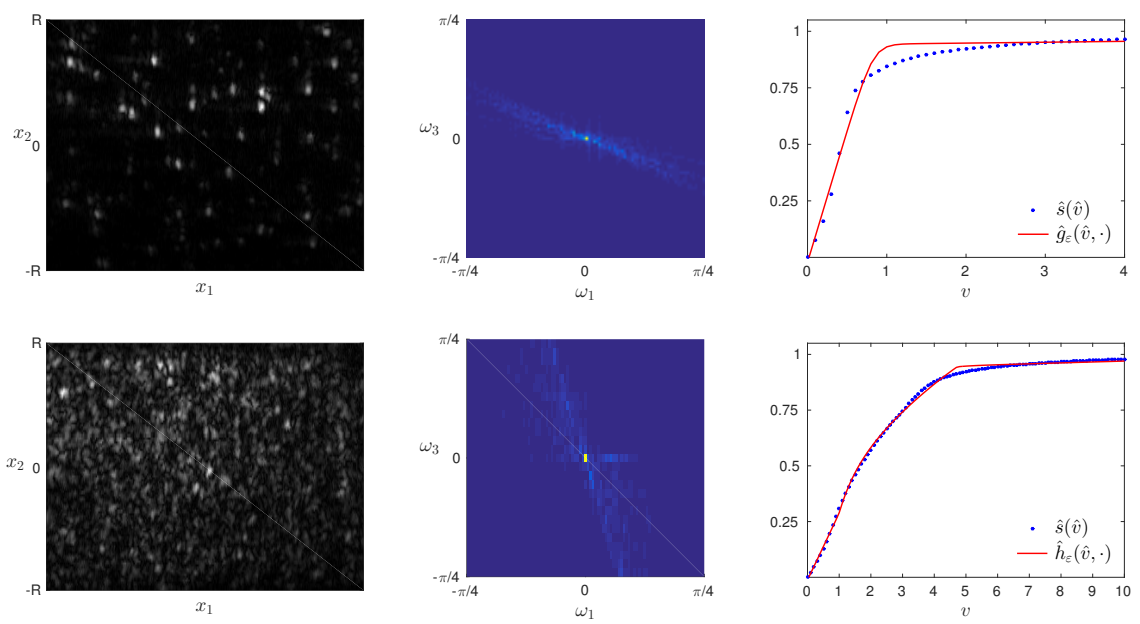

Figure 4.5: First row. Real air-bubbles flow. Second row. Real microbubbles flow. Estimated parameter values are listed in Tables and 4 and 5.

\begin{tabular}{|c|c|c|c|c|c|c|c|}
\hline & $\alpha$ & $\beta$ & $\delta$ & $\phi_{1}$ & $\phi_{2}$ & $\xi_{2}=v_{m}$ & $v_{m}^{*}$ \\
\hline microbubbles flow & 0.459 & 0.152 & 0.203 & -0.104 & -0.044 & 4.723 & 4.777 \\
\hline
\end{tabular}

Table 5: Parameters and peak velocity estimate for the microbubbles flow shown in Figure 4.5. Similar to the synthetic sequences with a large peak velocity (cf. Fig. 4.4 and Table 2), we obtained accurate estimates relative to the independent measurement $v_{m}^{*}$.

\section{Conclusion}

We presented a novel method for globally estimating pipe flows from plane wave ultrasound image sequence data. The method is based on a random particle model of the image sequence that is driven by the unknown flow. Applying the Fourier transform results in a piecewise linear model of the spectral support in the Fourier domain. Given a real image sequence, numerically fitting this model enables us to estimate the spectral support and in turn the peak velocity.

Attractive features of our method include: (a) It works directly on given image sequence data without the need of image preprocessing. (b) Since it is a global method, parameter estimation effectively copes with noisy scenarios that are encountered in real applications. (c) The numerical computations are simple enough to render realtime implementations feasible. 


\section{Acknowledgment}

The author would like to thank Jason Voorneveld from the Department of Biomedical Engineering, Thorax Center, Erasmus MC, Rotterdam, The Netherlands, for providing the real data and the fruitful discussions about ultrasound techniques. The author also acknowledges the intellectual contribution of Prof. Dr. Christoph Schnörr and Prof. Dr. Stefania Petra from Heidelberg University.

\section{References}

[BGPS15] E. Bodnariuc, A. Gurung, S. Petra, and C. Schnörr, Adaptive Dictionary-Based Spatio-temporal Flow Estimation for Echo PIV, Proc. EMMCVPR, Lecture Notes in Computer Science, vol. 8932, Springer, 2015, pp. 378-391.

[BMR00] E. G. Birgin, J. M. Martinez, and M. Raydan, Nonmonotone Spectral Projected Gradient Methods on Convex Sets, SIAM Journal on Optimization 10 (2000), no. 4, 1196-1211.

[BPPS16] E. Bodnariuc, S. Petra, C. Poelma, and C. Schnörr, Parametric Dictionary-Based Velocimetry for Echo PIV, Proc. GCPR, Lecture Notes in Computer Science, vol. 9796, Springer, 2016, pp. 332-343.

[BSPS16] E. Bodnariuc, M. Schiffner, S. Petra, and C. Schnörr, Plane Wave Acoustic Superposition for Fast Ultrasound Imaging, International Ultrasonics Symposium (IUS), IEEE, 2016.

[CPTP16] M. Correia, J. Provost, M. Tanter, and M. Pernot, 4D Ultrafast Ultrasound Flow Imaging In Vivo Quantification of Arterial Volumetric Flow Rate in a Single Heartbeat, Physics in Medicine \& Biology 61 (2016), no. 23, 48-61.

[FJ90] D. J. Fleet and A. D. Jepson, Computation of Component Image Velocity from Local Phase Information, International Journal of Computer Vision 5 (1990), no. 1, 77-104.

[Geo15] S. G. Georgiev, Theory of Distributions, Springer, 2015.

[Hee88] H. J. Heeger, Optical Flow Using Spatiotemporal Filters, International Journal of Computer Vision 1 (1988), no. 4, 279-302.

[KHS04] H. Kim, J. Hertzberg, and R. Shandas, Development and Validation of Echo PIV, Experiments in Fluids 36 (2004), no. 3, 455-462. 
$\left[\mathrm{LBE}^{+} 15\right]$ C. H. Leow, E. Bazigou, R. J. Eckersley, A. C. H. Yu, P. D. Weinberg, and M. X. Tang, Flow Velocity Mapping Using Contrast Enhanced High-Frame-Rate Plane Wave Ultrasound and Image Tracking: Methods and Initial in Vitro and in Vivo Evaluation, Ultrasound in Medicine and Biology 41 (2015), no. 11, 2913-2925.

[Poe17] C. Poelma, Ultrasound imaging velocimetry: a review, Experiments in Fluids 58 (2017), 1-28.

[RW09] R. T. Rockafellar and R. J.-B. Wets, Variational Analysis, 2nd ed., Springer, 2009.

[RWWK07] M. Raffel, C. E. Willert, S. T. Wereley, and J. Kompenhans, Particle Image Velocimery - A Practical Guide, Springer, 2007.

[TF14] M. Tanter and M. Fink, Ultrafast Imaging in Biomedical Ultrasound, IEEE Transactions on Ultrasonics, Ferroelectrics, and Frequency Control 61 (2014), no. 1, 102-119.

$\left[\mathrm{VKV}^{+} 16\right]$ J. Voorneveld, P. Kruizinga, H. J. Vos, F. J. H. Gijsen, E. G. Jebbink, A. F. W. van der Steen, N. de Jong, and J. G. Bosch, Native Blood Speckle vs Ultrasound Contrast Agent for Particle Image Velocimetry with Ultrafast Ultrasound - In Vitro Experiments, International Ultrasonics Symposium, IEEE, 2016.

Ecaterina Bodnariuc,

Mathematical Imaging Group,

Heidelberg University, Germany.

Email: ecaterina.bodnariuc@iwr.uni-heidelberg.de 